\title{
PAUL BROCKLEHURST: 'IT'S ABOUT OPTIMISING HOW MUCH HEALTH AND CARE WE CAN DELIVER FOR PATIENTS'
}

\author{
Paul Brocklehurst is currently a senior clinical lecturer, NIHR Clinician Scientist and \\ Honorary Consultant in Dental Public Health at the University of Manchester. From \\ mid-May 2015, Paul will move to a new role as the Director of the Clinical Trials Unit in \\ North Wales and Professor of Health Services Research at the University of Bangor.
}

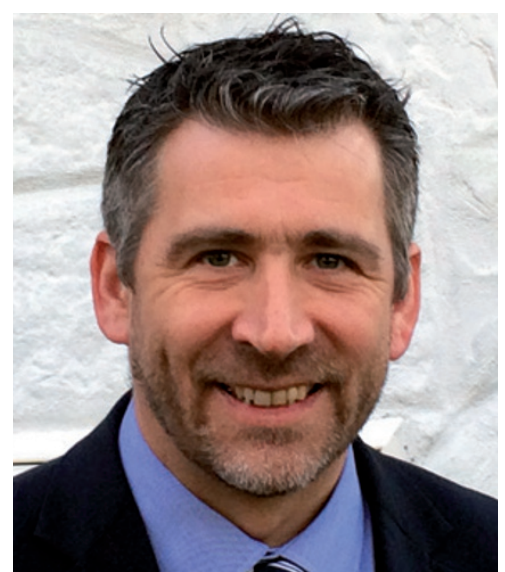

\section{What attracted you to work in the field of dentistry?}

I wanted to look for a career in healthcare as altruism was an important core value. Having received some dental treatment myself in my early teens, I realised that dentistry enabled me to combine the ability to care for people with an opportunity to develop a set of surgical skills and become exposed to new health technologies as they emerged. I was the first person in my family to go to university and the first to gain a qualification in healthcare, so I had no idea at the time whether it would turn out to be the right decision. Fortunately it was, and now I see my eldest daughter start her own journey in healthcare. She is midway through her training to fulfil her ambition to become a midwife.

\section{Why did you choose to specialise in dental public health?}

I'd been in practice in the NHS for quite some time and was very used to treating patients and managing my own practice from day-to-day. After about fifteen years or so, I got a bit bored of routine practice life and I wanted to push myself further academically. So, after undertaking all the Faculty of General Dental Practice examinations, I decided to study for a degree in psychology at the Open University. This really opened my eyes to the social sciences and exposed me to research as a creative process. It brought me into contact with a whole range of new ideas that took me beyond the relatively narrow confines of dentistry.

\section{'My role} within the NHS is to make sure that resources are used as wisely as possible'
Having completed my degree in psychology in 2004, I started to make some enquiries about whether I could start a PhD and approached Professor Paul Speight at the University of Sheffield, given his interest in oral cancer screening. Along with Sarah Baker, a registered health psychologist, we decided to look at the referral behaviours of general dental practitioners (GDPs) for oral cancer and potentially malignant disorders. This proved to be an interesting mixed-methods project, covering a range of academic disciplines and I thoroughly enjoyed the process. I did most of my $\mathrm{PhD}$ whilst continuing to run my own dental practice, which was a challenge and meant many weekends were taken up with academic work. This was fine as I enjoyed the whole process, but it did mean that my family had to be very patient!

I then sold my practice and went on to finish my $\mathrm{PhD}$ in the spring of 2009. This led me to look at academic dental public health (DPH) as a potential career route as I continued to enjoy taking a population approach to healthcare. DPH gives you the chance of asking some of the bigger questions and requires you to think strategically, which was something that built on my interest in the social sciences and what I had learnt from undertaking the $\mathrm{PhD}$. I started my specialist training later in 2009 at the University of Manchester and haven't looked back.

In 2011, I was fortunate enough to be awarded a prestigious National Institute for Health Research (NIHR) Clinician Scientist Award, which provided around one million pounds to explore role substitution in dentistry. This laid the foundation for further grant income and eventually my move to take up the post as the Director of the North Wales Clinical Trials Unit and Chair in Health Services Research. The University of Bangor has a strong reputation in implementation, healthcare sciences and psychology, so working to improve the health and well-being of the population through the design and conduct of complex pragmatic interventions is a great challenge for the future.

Imagine you are the Minister for Health for at least three years, what one thing would you most like to achieve?

That's an easy one in theory. My aim would be to reduce oral health inequalities and improve the equity of care in dentistry. I would base my approach on Maxwell's definition of quality - so ultimately this is about designing a service that responds to need, improves access, equity, effectiveness and efficiency.

My role within the NHS is to make sure that resources are used as wisely as possible but there are difficult decisions to make and DPH professionals are often confined by structural constraints or those that arise from the legal framework for NHS healthcare. Because of my experience in practice, it gives me a real advantage as I fully understand some of the tensions that dentists encounter. The need to improve our quality of care, as defined by Maxwell, is critically important, but it also has to make sense from a financial perspective as practices are businesses. For me it's all about aligning these 
DPH principles with the financial incentives in the contract and removing cultural or structural barriers that prevent change.

This raises the question as to whether the current contract is fit for service, moving forward. Adult dental health at a population level is improving quite remarkably, yet we still have intransigent disease in many young children and the need to design an appropriate service for older people is becoming increasingly important. By 2050, about half the population will be over fifty years of age and almost a quarter will be pensioners. As they age further, many older people will also retain some or all of their dentition at a time of decreasing independence, deteriorating diet and polypharmacy. This poses a range of issues for dentistry that we haven't fully explored yet. We need to understand what older people's oral health needs are, particularly as they lose their independence, and to examine how we might best manage them as they enter care or are cared for at home. We are also likely to see substantive health inequalities in this age group, a trend that we continue to see across the whole age range; the poorest still experience the highest burden of disease.

These changing oral health needs will have a knock-on effect on how we design our NHS dental services moving forward. I think there will be a need for greater stratification in the dental workforce, so that we can target these different cohorts in the population and encourage prevention. Until now, we have relied on an activity-based, feefor-service remuneration system to deliver the majority of our oral healthcare in the United Kingdom, but as the health of adult regular attenders improves further, we need to unlock the resources that are tied to the NHS Dental Contract and encourage dentists and their teams to become more communityfocused and provide leadership and creativity to address local oral healthcare problems. I have been involved in a number of projects across Greater Manchester where we have managed to align the payment system with DPH priorities for the region and we have seen real change in behaviour at a practitioner level, with dentists taking the lead in delivering oral health improvements locally. In one project, we managed to attract over 5,000 under five year old children to enter care for the first time within a two-month period and approaching half the practices across Greater Manchester wanted to actively engage. Moving towards a capitation-based or blended approach will help, but so will the innovative use of the dental workforce, population approaches to prevention, like targeted postal toothbrush and toothpaste schemes and national schemes like Designed to Smile and Childsmile.

\section{What do you think the role of the dentist will be in ten years' time?}

I think the role of the GDP will be to lead the local dental workforce team. If I was to make a prediction, I would say that the dental health of those that attend regularly will continue to improve, but health inequalities will persist. I think we need to look at innovative ways in the NHS of maintaining the health of our regular patients, whilst concomitantly addressing the oral health needs of our local communities to address the health inequalities I mentioned previously and respond to the future challenges of intransigent disease and changing demographics. I don't think we can do this with a 'one size fits all' approach. I do think we need to look at innovative ways of using the workforce, so that the skills of the dentist and the NHS resources that are inherently tied to their use, could be better utilised.

For me, the greater use of the whole of the dental team is paramount; care for routine adult patients, primary, secondary and some tertiary prevention could be undertaken by members of the team other than the dentist, with the dentist taking on the leadership role and the provision of more complex care. I think a 'systems' approach is needed to appropriately plan the future of the dental workforce based on future changes in demographics, population need, the level of service required to meet this need and the number and mix of clinicians that are required to provide this level of service. In short, a needs-based planning model.

'We need to
understand
what older
people's
health needs
are, particu-
larly as they
lose their
independence'

In the UK, we have the Bologna convention which increases the supply of dentists into the healthcare market from EU countries and there are also incentives in the educational system that lead to oversupply. The HEFCE tariff for training dentists is much higher than for other members of the dental team and dentists also attract a payment from the NHS for their training (SIFT), which others don't. This means that oversupply is likely to continue. Rather than training different members of the dental team to augment the activity of dentists, what could well happen is wage suppression amongst associate dentists, given this oversupply. This has happened in medicine.

I think we need to take a step back, understand what we need and design our educational and remuneration system to best fit the needs of our population, re-orientating our approach to focus on maintaining health and preventing disease across the age range and social gradient.

\section{Role substitution in primary dental care is the main focus of your research at the moment - what have you discovered?}

We have three ongoing studies. Two relate to investigating whether or not dental care professionals (DCPs) can screen for dental diseases in practice and whether they are safe as frontline healthcare workers (ie can they identify oral malignancy and potentially malignant disorders). We have found little difference, if any, between dentists and DCPs in the former study when screening for caries and periodontal disease. This work has subsequently been published in the Journal of Dental Research.

In the latter study, we saw more variation within the groups, than across the groups, which suggests that training is important for all clinicians. However, on the basis of this study, there appears to be no issue regarding the safety of using DCPs as frontline healthcare workers. This work will be published shortly in the British Dental Journal and hopefully will address the concerns raised by some in the profession about the more innovative use of DCPs in practice.

The third study is examining the productivity of dental teams and compares those that use DCPs 
and those that don't. This is being undertaken across England, Northern Ireland and Scotland. Analysis is still ongoing and we hope to publish the results next year.

The results of the first two studies suggest that DCPs could be used to maintain the oral health of routine low-risk patients in practice and this is the subject of a further grant application to the NIHR. This will be for a trial across England, Scotland and Wales to examine health outcomes for two new models of care, compared to usual practice.

\section{What other projects are you working on at the moment?}

We are beginning to look at how to design appropriate services for older people. We have just finished a research project that asked older patients what their priorities were in terms of oral health and what they thought was important in terms of service design in the future. This is known as a 'priority setting partnership' and encourages co-design. Maintaining function and reducing pain were both considered important, but so was the impact of oral health on the ability of older people to interact socially. This study has just been published

One of the things that's very interesting about this particular area of work is the ethical tensions it raises. With children there is an ethical imperative to measure how much need there is and then to design a service to address this need. But with older people there is an interesting dilemma between the need that we measure (what we'd term objective need) and their expressed need (from the patient's own perspective). As people become increasingly dependent on others for care, there can be a tension between objective and expressed need. It may not be appropriate to intervene in all cases and research here is very much in its infancy.

\section{What are your thoughts on direct access?}

We've undertaken quite a lot of research in the Netherlands on direct access, as it was introduced as part of a comprehensive set of changes to the legal, educational and contractual system in 2006. Most good practice is associated with clinicians working together in larger multi-surgery practices, with dentists being happy to refer and receive referrals. In these practices, hygiene-therapists are often used to screen routine patients and they take on the bulk of simple restorative care.

I think this model of integrated care has the most value moving forward from the perspective of efficiency and cost-effectiveness, where all of the dental team are contributing to the care of the patient. Where cases are too complex for the hygiene-therapist, they can be referred on within the practice, without too much disruption for the patient. The independent model raises more challenges and also requires dentists to continue to refer.

Interestingly, all our research over the past five years has shown high social acceptability for care by DCPs, with very little exception.

\section{From your experience, how does dentistry in the UK compare with that in other countries?}

I believe in a publicly funded healthcare system. I think it is the 'right' approach to healthcare and on balance, I think we do things well here. As I spoke about already, there is a need to stratify our approach moving forward to target at-risk groups, reduce health inequalities and improve the equity of care. We need to move away from a remuneration system that rewards activity to one that rewards the production of health across the age range and social gradient. I also think that local and national population health schemes are important. When you spend time in the United States and other private healthcare markets you realise how fortunate we are to have a publicly funded system. It gives us the ability to plan at a strategic level 'up-stream' and introduce population measures to address health, rather than simply relying on individual 'down-stream' interactions. We are also the envy of many academics in public health and health services research.

In the NIHR, we have one of the largest funding bodies in applied health to encourage research and the supportive infrastructure necessary to robust and rigorous evaluation. A lot of other countries don't necessarily have that level of funding for health service research, so we are very fortunate to have access to these funds to drive the quality of the NHS forward.

\section{What is your advice for students looking to specialise in dental public health?}

Ideally, they should try to get as much experience in as many different clinical settings as they can. This way they can understand some of the pressures that dentists face. I personally would also advocate an academic career in dental public health.

We are very fortunate in the UK in that we have a very good research infrastructure through the NIHR for DPH. This means there are a lot of funding opportunities and fellowship schemes that you can apply for. The latter provides a formal pathway to enable young researchers to gain clinical academic training. It is also important to be steered and supported by senior academics. I have been very fortunate to have had the opportunity to have received rigorous academic training from Professors Paul Speight and Sarah Baker at a doctoral level, and
Professor Martin Tickle at a postdoctoral level. Together with key NHS peers and colleagues, this has made a substantive difference to the quality of my training.

I certainly would not choose any other job. Being a clinical academic is so varied that no one day is the same. You can be talking with policy makers one day, engaged in research on another and working with patients the next.

\section{I suspect you travel frequently for your work; do you have a favourite place to visit?}

One of my favourite places is definitely the Netherlands because the Dutch people that I have met have been very generous in spirit. I have spent quite a lot of time over there and Utrecht in particular is very beautiful. It is a lot smaller than Amsterdam, but still has all the canals and great architecture. I also like the Western Seaboard of North America, particularly Vancouver and Seattle. The people are more relaxed and there are also many good vineyards and micro-breweries.

Since 2014, I have lived in North Wales where we have a smallholding. My wife and I enjoy taking care of our animals and we also love outdoor pursuits. Living close to both the sea and mountains means that returning home from abroad is always an easy process! We have two rare-bread Shetland sheep (Gwen and Nessa), along with some hens and ducks. We also have a sheepdog called Jackson. One of the male ducks has fallen in love with the dog. Jackson will lie on his back and get preened by this duck. I've never seen anything like it!

I am now able to combine a busy academic life, within a bustling clinical trials unit, with spending time out in the paddock. It helps put life into a unique perspective.

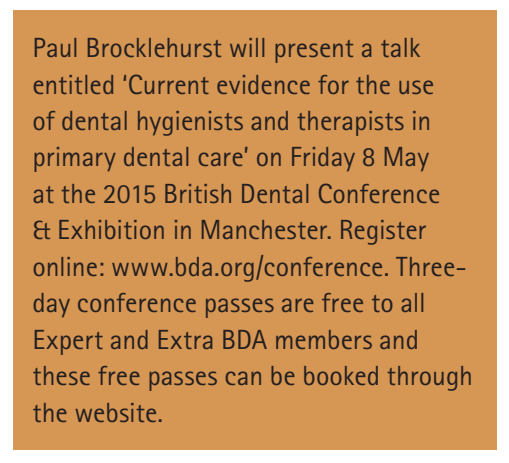

